\title{
CORRESPONDENCE \\ Changes in the humoral immunity response in SARS-CoV-2 convalescent patients over 8 months
}

\author{
Pai Peng ${ }^{1}$, Jie Hu${ }^{1}$, Hai-jun Deng (D) ${ }^{1}$, Bei-zhong Liu ${ }^{2}{ }^{2}$, Liang Fang ${ }^{2}$, Kai Wang ${ }^{1}{ }^{1}$, Ni Tang (D) and Ai-long Huang (D) \\ Cellular \& Molecular Immunology (2021) 18:490-491; https://doi.org/10.1038/s41423-020-00605-4
}

\begin{abstract}
Many countries around the world have seen a sharp rise in COVID-19 cases since the beginning of October due to the second wave of the pandemic. A decline in the antibody response to severe acute respiratory syndrome coronavirus 2 (SARS-CoV-2), which was reported exclusively in the early month, increases the risk of reinfection for convalescent individuals. There is a current need to follow the maintenance of specific antibodies against SARS-CoV-2.

Twenty patients who had recovered from COVID-19 were included in our cohort. Blood samples were obtained in February and October, corresponding to a median of 25 (range 5-33 days) and 230 (range 221-248 days) days after symptom onset (Fig. 1a). Enzyme-linked immunosorbent assay was performed to evaluate the presence of anti-SARS-CoV-2 spike (S) receptor-binding domain (RBD) lgG over 8 months. A preliminary positive cutoff was set with the mean value of negative controls above 3 standard deviations. ${ }^{1}$ Neutralizing antibodies (NAbs) were measured by pseudovirus-based assays associated with two SARS-CoV-2 strains (S-D614 and S-G614) in 293T-ACE2 cells. The $50 \%$ inhibitory dose $\left(\right.$ ID $\left._{50}\right)$ was calculated as the NAb titer.

Of the convalescent patients, there were 9 women and 11 men. The median age was 51.5 years (range 45-65). Except for two cases with severe symptoms, $90 \%$ of the infected patients had mild symptoms (Supplementary Table 1).

In all 20 participants, antibodies against the SARS-CoV-2 spike RBD decreased from a mean $\mathrm{OD}_{450}$ value of 1.78 (range $0.55-2.72$ ) to 0.38 (range $0.15-1.01$ ) over 8 months. When the $\mathrm{OD}_{450}$ value was $<0.26$, the specimen was considered seronegative. At follow-up time point 2 (in October), the lgG level of five participants (25\%) had became negative (Fig. 1b). A similar decline was observed in the pseudovirus neutralization assay. Indeed, NAb titers decreased
\end{abstract}

from a mean $I_{50}$ value of 836.55 (range 263-1160) to 170.30 (range $33-365)$. Among them, the NAb titers of three participants (15\%) were lower than the threshold at 8 months after symptom onset (Fig. 1c). Moreover, NAb titers correlated significantly with IgG levels $(p<0.001)$ (Fig. 1d). The cross-protective role of NAbs at 8 months after symptom onset was evaluated by a pseudovirus-based neutralization assay using SARS-CoV-2 S-G614, which is currently the dominant strain worldwide. The NAb titers against the S-G614 mutant pseudovirus of five participants (25\%) decreased below the threshold. Moreover, there was a statistically significant difference in the neutralizing efficacy of convalescent plasma against SARSCoV-2 S-D614 and S-G614 mutant pseudoviruses (Fig. 1e).

Herein, we report changes in the humoral immunity response in SARS-CoV-2 convalescent patients over 8 months. In agreement with previous follow-up studies within a shorter time frame, declines in both IgG and NAb were observed. ${ }^{1-3}$ Furthermore, the better significant correlation between lgG and NAb levels in February than in October indicates that the anamnestic immune response and other protective immunity should be evaluated within the context of low levels of NAbs. ${ }^{4}$

Facing the challenge of the second wave of SARS-CoV-2 infection, the risk of reinfection among convalescent patients by the currently dominant strain (SARS-CoV-2 S-G614) is worth considering. Weaker neutralizing activity against the S-G614 mutant pseudovirus has been demonstrated. In two samples, NAb titers even quickly decreased from 1:99 or 1:122 to near the limit of detection. This might be a warning about the possible loss of protective capacity for convalescent plasma with lower titers against the SARS-CoV-2 S-G614 variant, similar to the reinfection case reported in Hong Kong. ${ }^{5}$ Therefore, more data about the longevity of humoral immunity are needed to evaluate the effectiveness of herd immunity.

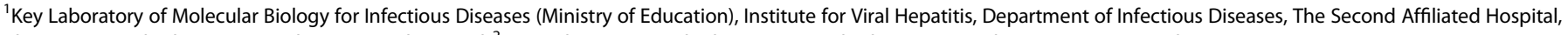
Chongqing Medical University, Chongqing, China and ${ }^{2}$ Yong-Chuan Hospital, Chongqing Medical University, Chongqing 402160, China Correspondence: Ai-long Huang (ahuang@cqmu.edu.cn) or Ni Tang (nitang@cqmu.edu.cn) or Kai Wang (wangkai@cqmu.edu.cn)

These authors contributed equally: Pai Peng, Jie Hu
}

Received: 13 November 2020 Accepted: 19 November 2020

Published online: 8 January 2021 
a

Sample collection timeline

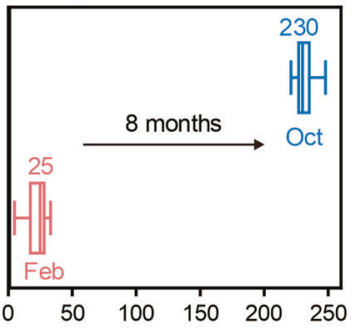

Days after symptom onset b

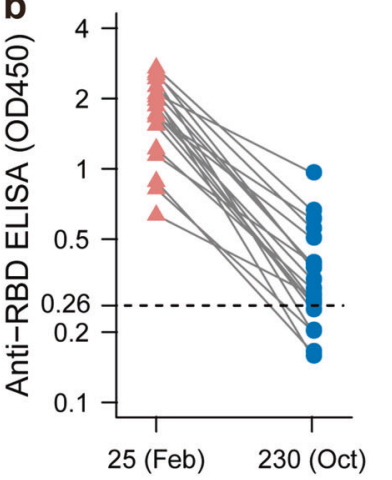

Days after symptom onset
C

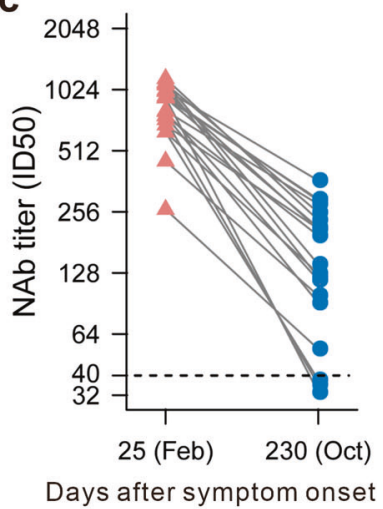

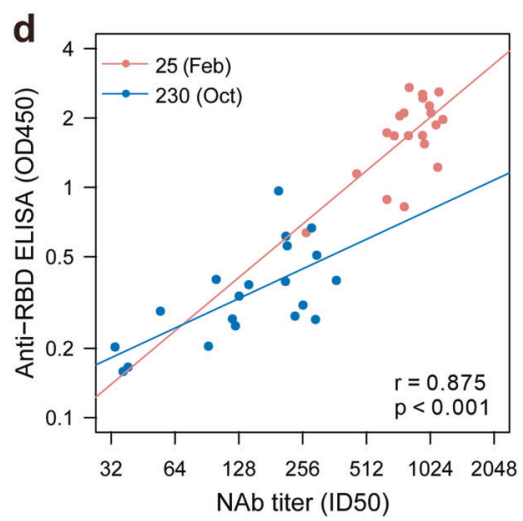

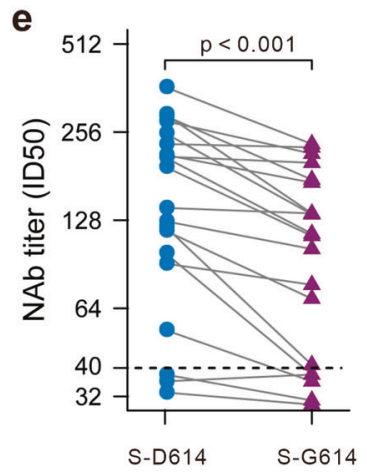

Fig. 1 Maintenance of the humoral response to SARS-CoV-2 in convalescent patients over 8 months. a Blood samples were collected in February and October. Enzyme-linked immunosorbent assays (ELISAs) (b) and pseudovirus-based neutralizing assays (c) were performed to detect lgG levels and neutralizing antibody (NAb) titers against SARS-CoV-2. The thresholds of detection were 0.26 for the OD $\mathrm{D}_{450}$ value and 1:40 for the ID $\mathrm{D}_{50}$. d Correlation of IgG and NAb levels. e Neutralizing activities of convalescent plasma against SARS-CoV-2 S-D614 or S-G614 mutant at 8 months after symptom onset

\section{ACKNOWLEDGEMENTS}

We acknowledge funding support from the Key Laboratory of Infectious Diseases (CQMU, 202005), the Emergency Project from the Science \& Technology Commission of Chongqing (cstc2020jscx-fyzx0053), the Emergency Project for Novel Coronavirus Pneumonia from Chongqing Medical University (CQMUNCP0302), the Leading Talent Program of CQ CSTC (CSTCCXLRC201719), and a Major National Science \& Technology Program grant (2017ZX10202203) from the Science \& Technology Commission of China.

\section{AUTHOR CONTRIBUTIONS}

A.H., N.T., K.W., P.P. and J.H. developed the conceptual ideas and designed the study. P.P. and J.H. performed the experiments. B.L. and L.F. provided the samples. H.D. performed the statistical analysis. All authors provided scientific expertise and interpreted the data. P.P. drafted the manuscript. All authors contributed to critical revision of the manuscript for important intellectual content. All authors reviewed and approved the final version of the report.

\section{FUNDING}

Our work has received funding support from the Key Laboratory of Infectious Diseases (CQMU, 202005), the Emergency Project from the Science \& Technology Commission of Chongqing (cstc2020jscx-fyzx0053), the Emergency Project for Novel Coronavirus Pneumonia from Chongqing Medical University (CQMUNCP0302), the Leading Talent Program of CQ CSTC (CSTCCXLJRC201719), and a Major National Science \& Technology Program Grant (2017ZX10202203) from the Science \& Technology Commission of China.

\section{ADDITIONAL INFORMATION}

The online version of this article (https://doi.org/10.1038/s41423-020-00605-4) contains supplementary material.
Competing interests: The authors declare no competing interests.

\section{REFERENCES}

1. Ripperger, T. J. et al. Orthogonal SARS-CoV-2 serological assays enable surveillance of low prevalence communities and reveal durable humoral immunity. Immunity https://doi.org/10.1016/j.immuni.2020.10.004 (2020).

2. Patel, M. M. et al. Change in antibodies to SARS-CoV-2 over 60 days among health care personnel in Nashville, Tennessee. JAMA https://doi.org/10.1001/ jama.2020.18796 (2020).

3. Wang, K. et al. Longitudinal dynamics of the neutralizing antibody response to SARS-CoV-2 infection. Clin. Infect. Dis. https://doi.org/10.1093/cid/ciaa1143 (2020).

4. Chen, Y. et al. Quick COVID-19 Healers Sustain Anti-SARS-CoV-2 Antibody Production. Cell https://doi.org/10.1016/j.cell.2020.10.051 (2020).

5. To, K. K.-W. et al. COVID-19 re-infection by a phylogenetically distinct SARScoronavirus-2 strain confirmed by whole genome sequencing. Clin. Infect. Dis. https://doi.org/10.1093/cid/ciaa1275 (2020).
Open Access This article is licensed under a Creative Commons Attribution 4.0 International License, which permits use, sharing, adaptation, distribution and reproduction in any medium or format, as long as you give appropriate credit to the original author(s) and the source, provide a link to the Creative Commons license, and indicate if changes were made. The images or other third party material in this article are included in the article's Creative Commons license, unless indicated otherwise in a credit line to the material. If material is not included in the article's Creative Commons license and your intended use is not permitted by statutory regulation or exceeds the permitted use, you will need to obtain permission directly from the copyright holder. To view a copy of this license, visit http://creativecommons. org/licenses/by/4.0/.

(c) The Author(s) 2021, corrected publication 2022 